\title{
A NOVEL WORK FOR BIN PACKING PROBLEM BY ANT COLONY OPTIMIZATION
}

\author{
Nishant Kumar Singh ${ }^{1}$, Susobhan Baidya ${ }^{2}$ \\ ${ }^{I}$ Social Services and Amateur Researcher, Swarnim Foundation (NGO) \\ ${ }^{2}$ Assistant Professor, Heritage Institute of Technology (Kolkata)
}

\begin{abstract}
This paper presents Ant colony optimization metaheuristic solution for Bin packing problem (BPP). In the BPP, the aim is to combine a set of items into bins of a certain capacity so as to minimize the total number of bins. The bin packing is a well-known NP-hard combinatorial optimization problem. Only very little instances can be solved exactly, so for real-world problems we have to rely on heuristic solution methods. We are proposing an ant based optimization which was introduced by Dorigo in 1992, which in the past proved appropriate to solve many optimization problems. This ACO is inspired by the path-finding abilities of real ant colonies.It combines an artificial pheromone trail with simple heuristic information to stochastically build new solutions. This paper explores the ability of the ACO algorithm to balance between bins and objects in its decision making process. The solution quality and time to solution make ACO competitive as an optimization technique for NP-hard problems in which various factors such as cost and length are involved.[1][2]
\end{abstract}

Keywords: ACO, bin packing problem, NP- Problem

\section{INTRODUCTION}

Bin Packing Problem is the problem of packing objects of different weight into a finite number of equal-sized bins in a way that minimizes the number of bins used. Even though this problem seems to be rather simple it is a NP-hard combinatorial optimization problem, i.e., no procedure is able to solve each problem instance in polynomial time. There are so many real life application of Bin Packing Problem, such that Machine scheduling, Recording of all of a composer's music, where the length of the pieces to be recorded are the weights and the bin capacity is the amount of time that can be stored on an audio CD (about 80 minutes). Our algorithm is inspired by Bioinspired Ant Colony Optimization which was designed by scientist Dorigo and Gambardella (1997). We frame our algorithm in light of Ant Colony Optimization and compared the results with the work of Prof. Dr. Armin Scholl and Dr. Robert Klein that was based on Hybrid solution procedure of metaheuristic strategy tabu search and a branch and bound procedure.

\section{PROPOSED APPROACH}

\subsection{Mapping of Objects into a Weighted Graph}

In order to implement ACO algorithm, Bin Packing Problem is mapped into a non-directed asymmetric weighted graph. Graph can be represented by $G(v, e)$ where $v$ denotes set of vertices and e denotes set of edges. Weight associated with an edge will be the weight of the item to be selected. So if we are going from vertex $i$ to $j$ then weight will be the weight of item $j$.

\subsection{Solution Construction:}

Every ant from the group of ants starts their journey with an empty bin and set of the entire object to be placed in the bin. Each ant pick objects one by one and place them in the bin until none of the available object can fit into the current bin. Then current bin is closed and a new bin is opened.

The trail of pheromone $(i, j)$ represents the desire to have an $\mathrm{i}$ object and a $j$ object in the same bin. The probability so that ant ' $\mathrm{k}$ ' chooses a $j$ object as next object for the considered bin $b$, in a partial solution $s$ is

[1]

$$
\begin{array}{rlr}
P(i, j) & =\left\{\frac{\left[\tau_{b}(j)\right]^{\alpha}[\eta(j)]^{\beta}}{\sum_{g \in J_{k}(s, b)}\left[\tau_{b}(g)\right]^{\alpha}[\eta(g)]^{\beta}} \quad \text { if } j \in J_{k}(s, b)\right. \\
& =0 & \text { otherwise } \\
\tau_{b}(j) & =\left\{\frac{\sum_{i \in b}\left[\tau_{(i, j)]}\right.}{|b|} \text { if } b \neq\{\}\right. & \\
& =\quad & \text { otherwise }
\end{array}
$$

In this equation, $J_{k}(s, b)$ is the set of items that qualify for inclusion in the current bin $b$. They are the items that are still left after partial solution $s$ is formed, and are small enough to fit in bin $b ; \eta(j)$ is the item size $j ; \tau_{b}(j)$ is the pheromone value for an item size $j$ in a bin $b$. It is the sum of all the pheromone values between item size $j$ and the item sizes $i$ that are already in bin $b$, divided by the number of items in $b$ for normalization. If $b$ is empty, $\tau_{b}(j)$ is set to $1 ; \alpha$ and $\beta$ are the parameter that 
define the relative importance of the heuristic information as opposed to the pheromone value.

\subsection{Updating of Pheromones}

After all ants have constructed their tour, the pheromone trails are updated. This is done by first lowering the pheromone values on all arcs by a constant factor, and then adding pheromone on the arcs the ant have crossed in their tours. Pheromone evaporation is implemented by. [1]

Where $0<\rho<=1$ is the pheromone evaporation rate. The parameter $\rho$ is used to avoid unlimited accumulation of the pheromone trails and it enables the algorithm to "forget" bad decision previously taken .In fact if an arc is not chosen by the ants, its associated pheromone values decrease exponentially in the number of iterations. After evaporation, all ants deposit pheromones on the arcs they have crossed in their tour [1]

$$
\tau_{i j} \leftarrow \tau_{i j}+\sum_{k=1}^{n} \Delta \tau^{k}
$$

Where $\Delta \tau^{k}$ is the amount of pheromone ant $\mathrm{k}$ deposits on the arcs it has visited. It is defined as follows:

$$
\Delta \tau^{\mathrm{k}}=\mathrm{f}\left(\mathrm{s}^{\mathrm{k}}\right)
$$

Where $f\left(s^{k}\right)$ is the fitness function which was proposed by Falkenauer and Delchambre in [3] and is given by:

$$
f\left(s^{k}\right)=\frac{\sum_{i=1}^{N}\left(\frac{F_{i}}{C}\right)^{m}}{N}
$$

Where:

$N$ is the number of bins used by an ant;

$F_{i}$ is total weight of placed objects in bin $i$;

$C$ is the maximum capacity of the bin;

$m$ is a parameter that defines the importance of the nominator.

(Note: $\mathrm{m}=2$ has been suggested as the optimal value by Falkenauer and Delchambre)

\section{The Heuristics}

1. Open an empty bin.

2. Add the heaviest item that still fits in the bin

3. Repeat step 2 until no item is left that is light enough to fit in the bin.

4. Go back to step 1 until all items are placed

\section{Pseudo Code}

initialize pheromone matrix by a little value repeat $\operatorname{ANTBIN}(K)$ for a finite number of iteration procedure ANTBIN $(\mathrm{k})$

begin

$\mathrm{i}=0$

$\operatorname{bin}=1$

repeat

move an ant from object $\mathrm{i}$

repeat

select any object $\mathrm{j}$ after object i by probability

equation (1)

if size of bin exhausted then bin ++

until all the object $\mathrm{s}$ are selected for packing

until $\mathrm{k}$ no. ants finish individual tour.

repeat

update pheromone $\tau_{\mathrm{ij}}$ for each ant's tour by equation (2) \& (3)

until $\mathrm{k}$ number of updation over

end

\section{EXPERIMENTAL RESULTS}

In order to implement ACO over BPP, standard object instances were considered and they were sourced from [6].

We compared our solution with the work of Dr. Armin Scholl, Dr. Robert Klein. [5]. For all experiments, parameters were set to the following values: $m=2, \tau_{0}=0.023330$ (small value), $\alpha=2$, $\beta=1$. Values for $\alpha, \beta$ were derived from an experiment where optimum results were compared for different values of $\alpha, \beta$. [Graph-1]

\section{SAMPLE RESULT}

For a considered problem instance [3] 'N1C1W1_A' with 50 object of different weights in the range of $[1,100]$ and bin capacity of 100 , the number of maximum iteration was set to 100 but Ants were observed to be converging at optimal solution i.e. 26 bins after 40 iterations.[Graph -2].

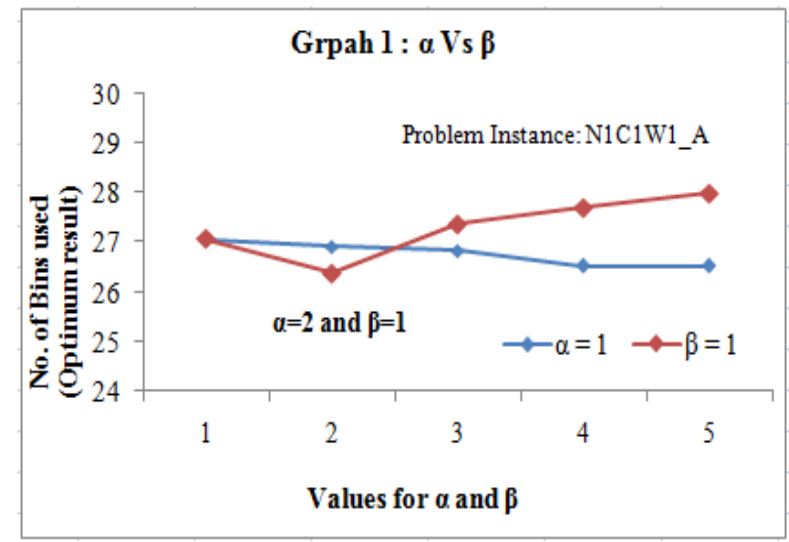




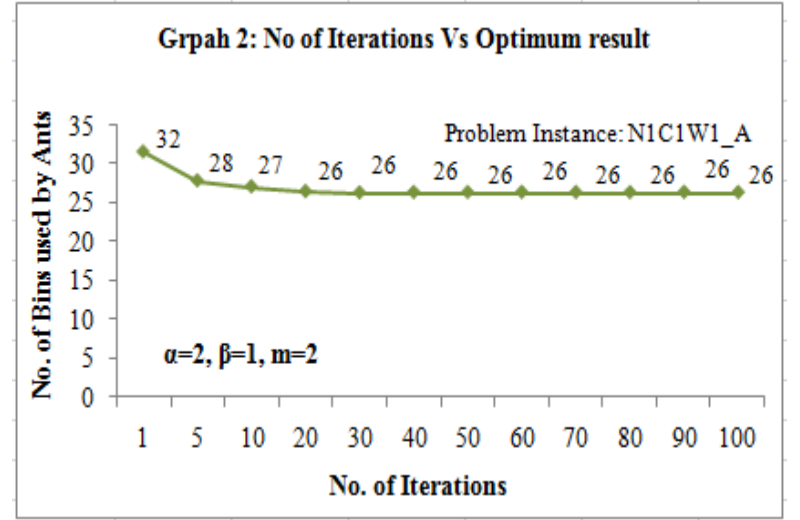

Convergence to optimal solution accelerates with increasing number of Ants as it leads to exploration of new solutions. [Grpah-3]

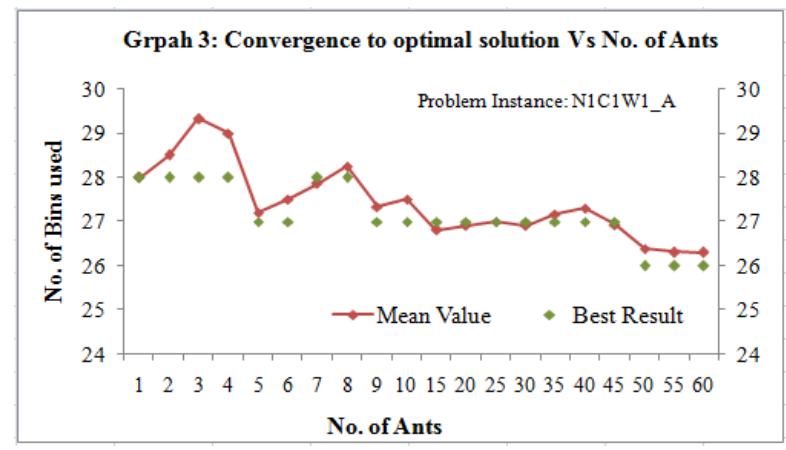

\section{Optimum Packing}

\begin{tabular}{|l|l|l|l|l|l|}
\hline $\begin{array}{l}\text { Bin } \\
\text { No. }\end{array}$ & Objects & $\begin{array}{l}\text { Bin } \\
\text { No. }\end{array}$ & Objects & $\begin{array}{l}\text { Bin } \\
\text { No. }\end{array}$ & Objects \\
\hline $\mathbf{1}$ & $61,27,7$ & $\mathbf{1 0}$ & 33,67 & $\mathbf{1 9}$ & 96 \\
\hline $\mathbf{2}$ & 46,42 & $\mathbf{1 1}$ & 24,74 & $\mathbf{2 0}$ & 99 \\
\hline $\mathbf{3}$ & 49,33 & $\mathbf{1 2}$ & 21,72 & $\mathbf{2 1}$ & 91 \\
\hline $\mathbf{4}$ & $28,40,28$ & $\mathbf{1 3}$ & $23,44,25$ & $\mathbf{2 2}$ & 96 \\
\hline $\mathbf{5}$ & $40,51,3$ & $\mathbf{1 4}$ & 67,30 & $\mathbf{2 3}$ & 92 \\
\hline $\mathbf{6}$ & 88,11 & $\mathbf{1 5}$ & 62,22 & $\mathbf{2 4}$ & 99 \\
\hline $\mathbf{7}$ & 87,10 & $\mathbf{1 6}$ & 76,20 & $\mathbf{2 5}$ & 92 \\
\hline $\mathbf{8}$ & $52,17,14,7$ & $\mathbf{1 7}$ & 30,69 & $\mathbf{2 6}$ & 85 \\
\hline $\mathbf{9}$ & 29,56 & $\mathbf{1 8}$ & 86,13 & & \\
\hline
\end{tabular}

\section{CONCLUSIONS AND FUTURE WORK}

From the above graphs we can conclude that the value of $\alpha=2$ and $\beta=1$ is optimal for BPP problem. The solution converges to its optimal point after 30 iterations and number of ants require for every iteration is 50 which is exactly number of objects in the BPP. Here in our algorithm we got optimal result 26 for the above instance, which is very close to our desire result [5]. We hope these tours can be encoded as superior genes for Genetic Algorithm for further refinement of results.

\section{REFERENCES}

[1]. Frederick Ducatelle "Ant Colony Optimisation for Bin Packing and Cutting Stock Problems", 2001

[2]. M. Dorigo, V. Maniezzo, and A. Colorni, "Ant System: Optimization by a colony of cooperating agents," IEEE Transactions on Systems,Man, and Cybernetics - Part B, vol. 26, no. 1, pp. 29-41, 1996.

[3]. Emanuel Falkenauer and Alain Delchambre. A genetic algorithm for bin packing and line balancing.In Proceedings of the IEEE 1992 International Conference on Robotics and Automation, Nice,France, May 1992.

[4]. M. Dorigo and T. St" utzle, Ant Colony Optimization. MIT Press, Cambridge, MA, 2004

[5]. Prof. Dr. Armin Scholl, Dr. Robert Klein, "http://www.wiwi.uni-jena.de/Entscheidung/binpp/ "

[6].

http://www.wiwi.unijena.de/Entscheidung/binpp/bin1dat.htm 